\title{
उMR
}

\section{Process of Bipolaris sorghicola invasion of host cells}

\author{
C. Peng ${ }^{1,2}$, T.T. $\mathrm{Ge}^{2}$, X.L. He ${ }^{2}$, Y.H. Huang ${ }^{2}$, Z.L. $\mathrm{Xu}^{2}$, D.Y. Zhang ${ }^{2}$, H.B. Shao ${ }^{2}$ and \\ S.W. Guo ${ }^{2}$
}

${ }^{1}$ State Key Laboratory for the Biology of Plant Disease and Insect Pests, Institute of Plant Protection, Chinese Academy of Agricultural Sciences, Beijing, China ${ }^{2}$ Provincial Key Laboratory of Agrobiology/Institute of Agrobiotechnology, Nanjing, Jiangsu, China

Corresponding author: S.W. Guo

E-mail: gogow12@126.com

Genet. Mol. Res. 15 (1): gmr.15016781

Received August 14, 2015

Accepted October 20, 2015

Published March 11, 2016

DOI http://dx.doi.org/10.4238/gmr.15016781

\begin{abstract}
Target leaf spot is a sorghum leaf disease caused by Bipolaris sorghicola, a species of fungus with a global distribution. In this study, we investigated the process by which $B$. sorghicola invades cells of barley, onion, Arabidopsis thaliana species, and sorghum. The results showed that within $8 \mathrm{~h}$ of coming into contact with host cells, the hyphal ends of $B$. sorghicola expand and form a uniform infective penetration pegboltlike structure; a primary infection mycelium can be formed inside host cells within $24 \mathrm{~h}$ after contact, which can infect closed cells after $48 \mathrm{~h}$. A mycelium can grow within the gap between cells and form infective hyphae. The pathogen infection process was the same in different host cells. $B$. sorghicola can affect root cells through soil infection, indicating that it may also have characteristics of soil-borne pathogens.
\end{abstract}

Key words: Bipolaris sorghicola; Hyphae; Infection process 


\section{INTRODUCTION}

Sorghum is a globally important cereal crop with resistance to various stresses (such as drought, salinity, and poor soil) and strong heterosis; it is an important specialty crop and coarse cereal, with high utilization value (Palmer, 1992). However, sorghum production is affected by diseases and pests.

Sorghum target leaf spot is a globally important fungal disease caused by Bipolaris sorghicola. The disease was first discovered in 1939 on a variety of sudangrass in Georgia, USA; it was subsequently found in the sorghum-growing regions of Cyprus, India, the Sudan, Japan, and Venezuela. Target leaf spot disease has become one of the major foliar diseases of sorghum and can cause a $50 \%$ reduction in production during a serious outbreak. Not only does $B$. sorghicola infect sorghum, but it is also the pathogen in a variety of crop diseases, such as rice brown spot (Moriwaki et al., 2004), wheat root rot (Nizam et al., 2012), and maydis leaf blight (Gogoi et al., 2014).

During the course of a disease, fungal pathogens experience a series of growth stages and metabolic pathways, which are collectively known as the disease cycle. For example, the disease cycle of the rice blast fungus is divided into the following five stages: the spores become attached to the host surface; the spores differentiate into germ tubes and appressoria; each appressorium produces an invasion nail to penetrate the stratum corneum and epidermal cell wall; the secondary mycelia are formed and grow within the host cell, infect neighboring epidermal cells, and enter the mesophyll; and finally, after 5-7 days, symptoms appear and new spores, which can re-infect the host, are generated and released from the lesion spots (Hamer et al., 1988; Kankanala et al., 2007). Throughout the disease cycle, the most interesting aspect is the process by which the fungus infects the host cells. The process of infection of the sorghum target leaf spot pathogen has rarely been reported. In this paper, we investigated the process by which the sorghum target leaf spot pathogen infects barley, onion, Arabidopsis, and sorghum cells to provide a theoretical basis for understanding and preventing the disease.

\section{MATERIAL AND METHODS}

\section{Material}

B. sorghicola strains were kindly donated by Professor Xu Xiude from the Plant Protection Department at the Liaoning Academy of Agricultural Sciences.

\section{Experimental methods}

\section{Strain culture}

B. sorghicola strains were preserved in potato dextrose agar medium at $28^{\circ} \mathrm{C}$ in the dark for approximately 3 days. We then divided the potato dextrose agar liquid medium into small portions, centrifuged them at $150 \mathrm{rpm}$ and $28^{\circ} \mathrm{C}$ for 2 days, and set them aside.

\section{Barley epidermal infection experiments}

We clipped barley leaves that had been growing for approximately 1 week and put them on Petri dishes containing moistened filter paper with their posterior sides facing up. The above- 
mentioned cultivated mycelial pellets were washed three times with sterile water. We secured the mycelium ball on the epidermis of the barley leaf posterior sides and cultured at $28^{\circ} \mathrm{C}$ in the dark for 8,24 , and $48 \mathrm{~h}$. We then tore the barley epidermis and prepared the sections for photographic recording (Olmpus CX31, Japan).

\section{Onion epidermal infection experiments}

An onion was cut into $3 \times 2$-cm pieces. We mounted the onion epidermis on a slide by using sterile water to preserve moisture. The mycelium ball procedure was the same as that described above in the section entitled "Barley epidermal infection experiments". The onion sections were cultured at $28^{\circ} \mathrm{C}$ in the dark for 8,24 , and $48 \mathrm{~h}$ and prepared for photographic recording.

\section{Arabidopsis and sorghum leaf infection experiments}

The mycelium ball procedure was the same as that described above in the section entitled "Barley epidermal infection experiments". We subjected inoculated samples that had been cultured for 8, 24, and $48 \mathrm{~h}$ to Trypan blue (Kelong Chemical, ChengDu, China) staining to selectively color dead cells according to the method described by Frye and Innes (1998).

\section{Root colonization experiment}

After the sorghum seed germinated for 7 days, cuted one piece of mycelia, and inoculated it in the soil around the sorghum root, kept moisture in $28^{\circ} \mathrm{C}$ for 3 days, then recorded by microscope photograph.

\section{RESULTS}

\section{Process of barley epidermis invasion by $B$. sorghicola}

When the mycelial pellets of $B$. sorghicola were cultured with the barley leaves for $8 \mathrm{~h}$, a small number of infection hyphae were found in the epidermal cells of the barley; after $24 \mathrm{~h}$, infection hyphae were visible in the cells, but extension was not obvious; after $48 \mathrm{~h}$, the cells were filled with infection hyphae, which were invading the closed cells (Figure 1).

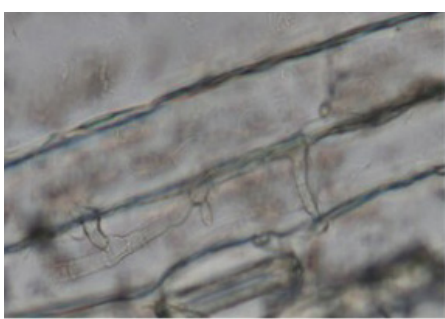

$8 \mathrm{~h}$

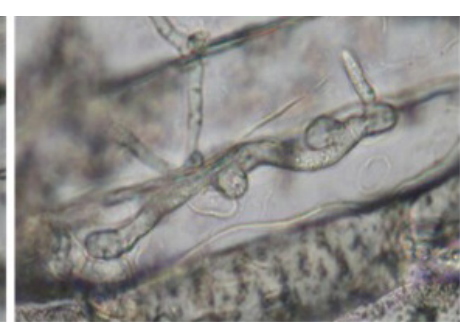

$24 \mathrm{~h}$

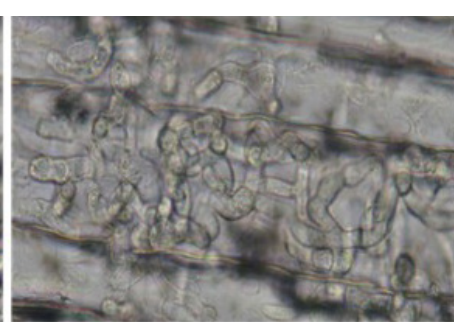

$48 \mathrm{~h}$

Figure 1. Invading process of Bipolaris sorghicola in barley epidermis. 


\section{Process of onion epidermis invasion by $B$. sorghicola}

Onion epidermal cells are readily available and easy to monitor, and are therefore very useful for the investigation of pathogens. However, the pathogenicity of $B$. sorghicola in onions has not been reported. In this study, we inoculated $B$. sorghicola mycelia into onion epidermal cells to investigate the invasion process. The results showed that after $8 \mathrm{~h}$ of inoculation, the ends of the mycelia expanded and attached to the surface of the onion epidermal cells. Hyphae existed in the intercellular spaces. After $24 \mathrm{~h}$, the infection hyphae could be clearly seen in the intracellular spaces. The hyphae that grew in the gap between the cells were also able to invade surrounding cells. After $48 \mathrm{~h}$, the infecting mycelia had covered the entire cell (Figure 2), indicating that the sorghum target leaf spot pathogen can infect onion epidermal cells via two pathways: the cell surface and the gaps between the cells.

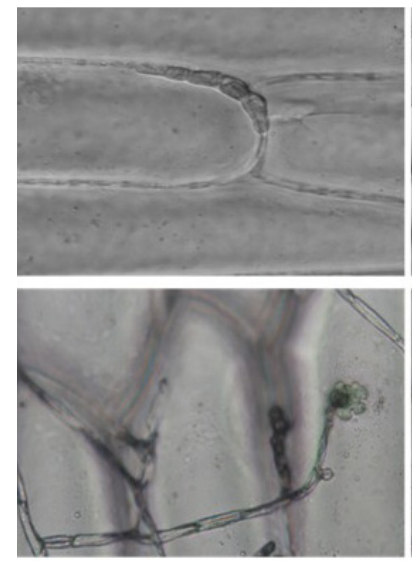

$8 \mathrm{~h}$

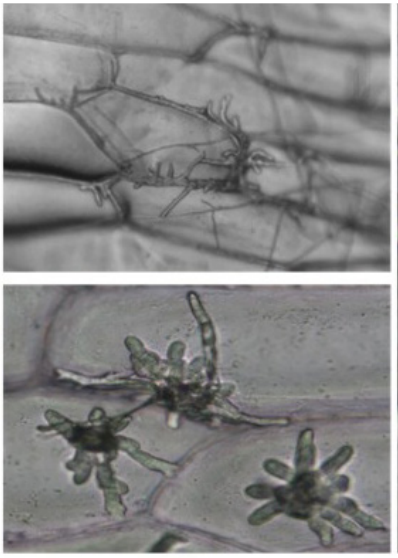

$24 \mathrm{~h}$

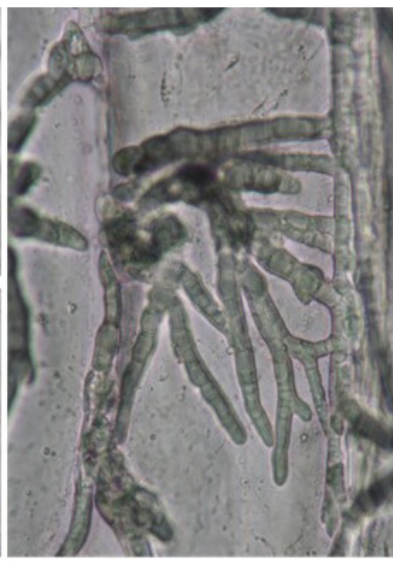

$48 \mathrm{~h}$

Figure 2. Invading process of Bipolaris sorghicola in onion epidermal.

\section{Infection of sorghum target spot pathogen in sorghum and Arabidopsis leaf epidermis}

Owing to the difficulty in obtaining Arabidopsis and sorghum epidermal cells, in the present study we used Trypan blue staining to investigate infection by the sorghum target spot pathogen in sorghum and Arabidopsis leaf cells. The results showed that the infection process of the target leaf spot pathogen in sorghum and Arabidopsis leaves was consistent with that in barley and onion epidermis cells, and the pathogen can enter the interior of the cells from the cell surface and from the gaps between the cells (Figures 3 and 4), indicating that the sorghum target leaf spot pathogen also has pathogenicity in Arabidopsis.

\section{Infection of sorghum target spot pathogen in sorghum root cells}

The route taken by pathogenic fungi when infecting plants is not necessarily through the leaf epidermal cells. In addition to leaf spot, rice blast fungus can cause disease in the roots of rice plants by infection through the soil. In this study, we also investigated infection by the sorghum 
target leaf spot pathogen in sorghum root cells. Figure 5 shows that the sorghum target leaf spot pathogen can contact the root cells through the soil and successfully enter the interior of the cells. Sorghum root cells in contact with mycelia produce purple substances; this change did not occur in neighboring cells without contact with mycelia, suggesting that the purple substances may be associated with disease resistance in sorghum.

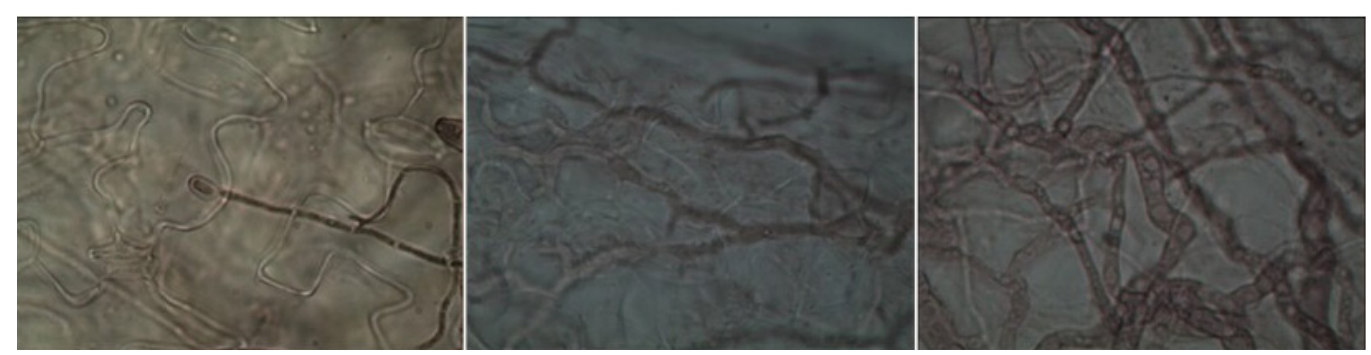

Figure 3. Infection of sorghum target spot pathogen in Arabidopsis leaf epidermis.

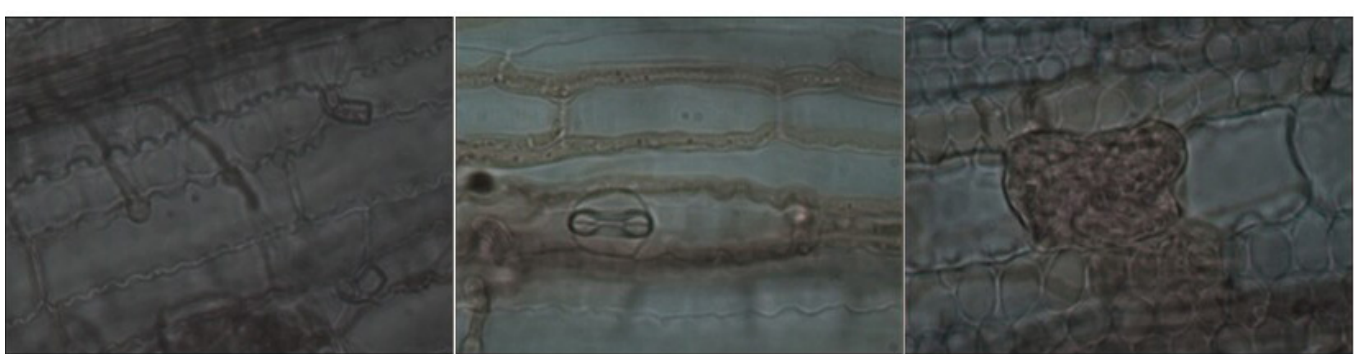

Figure 4. Infection of sorghum target spot pathogen in sorghum leaf epidermis.

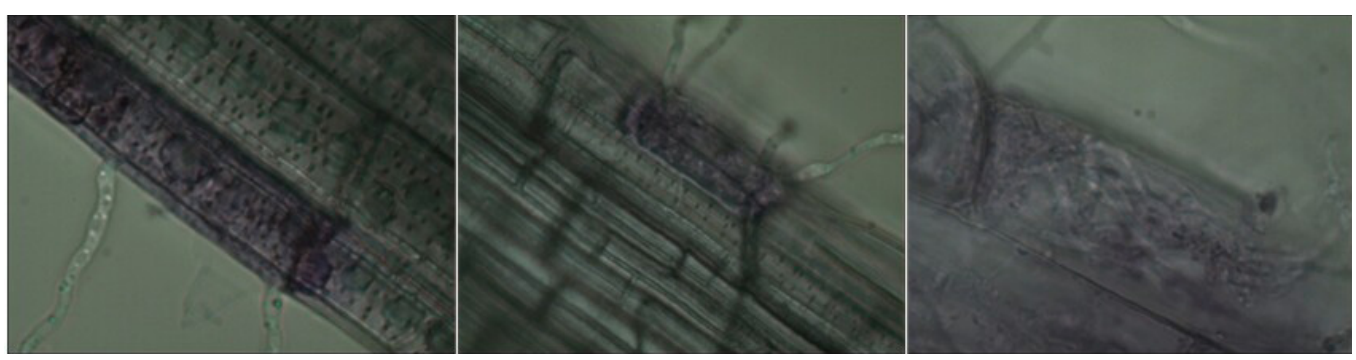

Figure 5. Infection of sorghum target spot pathogen in sorghum root cells.

\section{DISCUSSION}

Understanding the disease cycle of pathogens and the infection process within the host cell can provide a theoretical basis for disease control. By studying the infection process of the sorghum target leaf spot pathogen in barley, onion, Arabidopsis, and sorghum cells, we found that the temporal and spatial infection process of this pathogen was consistent in different plant cells; it can enter the interior of cells via the cell surface or the gaps between cells, and the mycelia that were in contact with the cell surface in the initial infection, which may be important structures for infec- 
tion, were swollen. Some reports have indicated that infection by $B$. sorghicola has no significant correlation with its appressoria (Lev et al., 1999). Therefore, in this study, we paid no attention to the development of appressoria when observing the process of infection adopted by the sorghum target leaf spot pathogen. At the same time, our paper is the first to describe the pathogenicity of sorghum target leaf spot pathogen in onions and Arabidopsis. Therefore, Arabidopsis, as a model dicot organism, can be used for interaction studies between the sorghum target leaf spot pathogen and plants. Our study objective presented several challenges: it is difficult to obtain sorghum and Arabidopsis leaf epidermal layer cells and the disease-causing capability of mutants is very important in the study of the gene function of pathogens. To solve these problems we exploited the fact that the sorghum target spot pathogen infection process in sorghum leaf cells is the same as that in onion and barley epidermal cells. Therefore, we selected onion and barley epidermal cells instead of sorghum epidermal cells to conduct our pathogenic experiments and observe cytological changes in the mutant infection process, thereby providing assistance for gene functional studies on the sorghum target leaf spot pathogen and $B$. sorghicola.

The main identifying characteristics of plant disease are the common symptoms arising from pathogen infection in specific tissues, such as leaf spots, root rot, and wilting plants. Thus, plant pathogens are habitually divided into leaf and soil-borne pathogens. In this experiment, we inoculated the sorghum target spot pathogen into the planting soil of sorghum; the results showed that the pathogen can contact sorghum roots through the soil and successfully infect cells, indicating that the target leaf spot pathogen of sorghum may also have characteristics of soil-borne pathogens. Therefore, in any disease prevention strategy, the roots should also be of concern. Moreover, we observed that purple substances were generated in the sorghum root cells when they came into contact with mycelia, suggesting that these substances may be associated with disease resistance in sorghum, which was consistent with the results reported by Liu et al. (2010). However, in sorghum leaves this phenomenon was not observed; this may be because these disease-related substances were eluted during Trypan blue staining and post-processing.

\section{Conflicts of interest}

The authors declare no conflict of interest.

\section{ACKNOWLEDGMENTS}

Research supported by the Open Research Fund Program of the State Key Laboratory of Virology of China (\#SKLOF201519), and the Jiangsu Agriculture Science and Technology Innovation Fund [CX(14)2001].

\section{REFERENCES}

Frye CA and Innes RW (1998). An Arabidopsis mutant with enhanced resistance to powdery mildew. Plant Cell 10: 947-956. http://dx.doi.org/10.1105/tpc.10.6.947

Gogoi R, Singh S, Singh PK, Kulanthaivel S, et al. (2014). Genetic variability in the isolates of Bipolaris maydis causing maydis leaf blight of maize. Afr. J. Agric. Res. 9: 1906-1913.

Hamer JE, Howard RJ, Chumley FG and Valent B (1988). A mechanism for surface attachment in spores of a plant pathogenic fungus. Science 239: 288-290. http://dx.doi.org/10.1126/science.239.4837.288

Kankanala P, Czymmek K and Valent B (2007). Roles for rice membrane dynamics and plasmodesmata during biotrophic invasion by the blast fungus. Plant Cell 19: 706-724. http://dx.doi.org/10.1105/tpc.106.046300 
Lev S, Sharon A, Hadar R, Ma H, et al. (1999). A mitogen-activated protein kinase of the corn leaf pathogen Cochliobolus heterostrophus is involved in conidiation, appressorium formation, and pathogenicity: diverse roles for mitogen-activated protein kinase homologs in foliar pathogens. Proc. Natl. Acad. Sci. U. S. A. 96: 13542-13547. http://dx.doi.org/10.1073/ pnas.96.23.13542

Liu H, Du Y, Chu H, Shih CH, et al. (2010). Molecular dissection of the pathogen-inducible 3-deoxyanthocyanidin biosynthesis pathway in sorghum. Plant Cell Physiol. 51: 1173-1185. http://dx.doi.org/10.1093/pcp/pcq080

Moriwaki A, Kihara J, Kobayashi T, Tokunaga T, et al. (2004). Insertional mutagenesis and characterization of a polyketide synthase gene (PKS1) required for melanin biosynthesis in Bipolaris oryzae. FEMS Microbiol. Letts. 238: 1-8.

Nizam S, Verma S, Singh K, Aggarwal R, et al. (2012). High reliability transformation of the wheat pathogen Bipolaris sorokiniana using Agrobacterium tumefaciens. J. Microbiol. Methods 88: 386-392. http://dx.doi.org/10.1016/j.mimet.2012.01.004

Palmer GH (1992). Sorghum-food, beverage and brewing potentials. Process Biochem. 27: 145-153. http://dx.doi. org/10.1016/0032-9592(92)87002-X 